\title{
Acetabular dysplasia and osteoarthritis of the hip in elderly white women
}

Departments of
Medicine and
Epidemiology,
University of
California at San
Francisco, San
Francisco, California,
USA
N E Lane
M C Nevitt
A Pressman
R Gore
Medical Research
Council
Environmental
Epidemiology Unit,
Southampton General
Hospital,
Southampton
C Cooper
Departments of
Medicine and
Epidemiology and
Preventive Medicine,
University of
Maryland School of
Medicine, and
Geriatric Service and
Gerontology Research
Education and Clinical
Center, Veterans
Affairs Medical
Center, Baltimore,
Maryland, USA
M Hochberg
Correspondence to:
Dr E Lane, Division of
Rheumatology, Box 0868,
University of California at
San Francisco, San
Francisco, California 94143 ,
USA.

Accepted for publication 30 June 1997

Nancy E Lane, Michael C Nevitt, Cyrus Cooper, Alice Pressman, Robert Gore, Marc Hochberg

\begin{abstract}
Objectives-To examine the association of acetabular dysplasia and osteoarthritis (OA) of the hip among elderly white women.

Methods-Pelvic radiographs from a sample of 165 white women aged 65 and above with radiographic hip $\mathrm{OA}$ and 88 white women aged 65 and above without radiographic changes of hip OA were read for evidence of acetabular dysplasia by a single trained investigator. Acetabular dysplasia was assessed using measurements of the centre edge angle and the acetabular depth, which are both reduced in this condition. Odds ratios for the association between acetabular dysplasia and hip OA were estimated using logistic regression analysis.
\end{abstract}

Results-Fourteen (3.4\%) hips had a centre edge angle $<25^{\circ}, 46(11.2 \%)$ hips had an acetabular depth of $<9 \mathrm{~mm}$, and 54 (13.2\%) hips had acetabular dysplasia defined as either of the above. Hips with OA had a small, but not statistically significant, increased prevalence of abnormal centre edge angle (odds ratio: $1.43 ; 95 \%$ confidence intervals: $0.46,4.46)$, abnormal acetabular depth $(1.47 ; 0.78$, $2.77)$ and acetabular dysplasia $(1.33 ; 0.74$, 2.40).

Conclusion-These results do not support the hypothesis that mild acetabular dysplasia accounts for a substantial proportion of hip $O A$ in elderly white women. A study with a much larger sample size would be required to rule out a weak association between dysplasia and hip OA of the magnitude actually observed in our study.

\section{(Ann Rheum Dis 1997;56:627-630)}

Osteoarthritis (OA) of the hip is an important cause of musculoskeletal disability in the elderly. ${ }^{1}$ Epidemiological studies suggest that hip OA is multifactorial in aetiology ${ }^{2}$ and have identified several risk factors for hip OA, including increasing age, overweight, trauma, and occupational physical activity. ${ }^{2-4}$ In addition, clinical studies of patients with hip OA suggest that structural abnormalities of the hip that are present at delivery or develop in childhood may result in accelerated or premature joint degeneration. ${ }^{5}$ Examples of these include congenital hip subluxation/dislocation, slipped capital femoral epiphysis, and Legg-Calve-Perthe's disease. However, the cumulative incidence of these disorders is too small to account for a large proportion of cases in the elderly. ${ }^{2}$ A more common structural abnormality that may predispose to hip OA is subclinical, or mild, acetabular dysplasia. Indeed, based on case series of patients with hip OA Murray, ${ }^{6}$ Stulberg and colleagues, ${ }^{7}$ and Harris ${ }^{8}$ estimated that between 25 and 40 per cent of cases of hip OA may be caused by subclinical acetabular dysplasia. In contrast, more recent studies of elderly British men $^{9}$ and women $^{10}$ and elderly Chinese men in Hong Kong $^{11}$ failed to find an association between indicators of subclinical dysplasia and hip OA in patients undergoing intravenous urograms whose pelvic radiographs were assessed for both hip OA and acetabular dysplasia. However, these studies were limited by relatively few cases of hip OA. The purpose of this study was to examine the association between indicators of acetabular dysplasia and hip OA assessed from pelvic radiographs in a large cohort of elderly white women participating in the Study of Osteoporotic Fractures.

\section{Methods}

SUBJECTS

Subjects were participants in the Study of Osteoporotic Fractures, a multicentre longitudinal cohort study of the risk factors for osteoporotic fractures in 9704 women. Participants were all age 65 and above at baseline examination and were recruited between September 1986 and October 1988 from population based listings in four areas of the United States: Baltimore, MD, Minneapolis, $\mathrm{MN}$, Portland, OR, and the Monongahela Valley near Pittsburg, PA. ${ }^{12}$ Non-white women were excluded because of their low incidence of hip fracture, as were women who were nonambulatory or who had undergone bilateral hip replacement.

RADIOGRAPHIC EVIDENCE OF OA

At the baseline examination, all women had a supine anteroposterior (AP) radiograph of the pelvis with the hips in 15-30 degrees of internal rotation. Pelvic radiographs of a consecutive sample of 5818 subjects were evaluated for hip OA. Hip radiographs were read for individual radiographic features of OA using an atlas illustrating the individual radiographic features to standardise the readings. ${ }^{13}$ Joint space narrowing (lateral and medial) and osteophytes (the largest of femoral or acetabular in both superior and inferior positions) were graded on a $0-3$ scale where $0=$ none, $1=$ mild, $2=$ moderate, and $3=$ severe or 
large, respectively. Subchondral sclerosis, cysts, and femoral neck deformity were graded as present or absent. Inter-rater reliability among readers was good to excellent: intra-class correlation $=0.82$ for narrowing and 0.74 for osteophytes; $\kappa=0.67$ for sclerosis and 0.70 for cysts. $^{12}{ }^{13}$ Definite joint space narrowing was present if the minimal joint space was $2.5 \mathrm{~mm}$ or less and the semi-quantitative grade was 2 or 3. Definite osteophytes correspond to grade 2 or 3 . Scores of $\geq 2$ were considered definite for narrowing or osteophytes because this cut off point had higher inter-rater reliability $(\kappa=0.71$ and 0.65 , respectively) than did scores $\geq 1$ ( $\kappa=$ 0.50 and 0.46 ).

A summary grade from $0-4$ for radiographic hip OA was defined for each hip in terms of the combination of radiographic features present. ${ }^{14}$ Grade 2 for radiographic hip OA required the presence of either definite osteophytes or joint space narrowing plus at least one other feature that included cysts or sclerosis. We considered grade 2 or greater to be positive for radiographic hip OA, and grade $3-4$ to be moderate to severe disease. Inter-rater reliability among the three readers for the summary grade was also good to excellent: intra-class correlation $=0.87$ for the $0-4$ score and $\kappa=$ 0.71 for a score of $0-1$ versus $2-4$. This summary grade has been shown to be highly correlated with self reported hip pain, ${ }^{15}$ decreased hip range of motion and hip abductor strength, ${ }^{16}$ higher bone mineral density, ${ }^{14}$ and the presence of hand OA. ${ }^{17}$

Pelvic radiographs were read by one of two primary readers (NEL, MCH). Radiographs with definite osteophytes or definite narrowing in either hip were also assessed by the second reader (97 or $19.5 \%$ of the films). Disagreement between the two readers in the summary grade assigned to either hip was resolved in a consensus reading of the pelvic film with both readers present (15 or $5.6 \%$ of films).

ACETABULAR MEASUREMENTS

Acetabular anatomy was assessed from a random sample of radiographs of 165 women with evidence of definite radiographic hip OA (summary grade of 2-4 in at least one hip) and 88 control women without hip OA. In this group of 253 pelvic radiographs, two right hips and six left hips could not be scored for OA because of either poor radiographic quality or a unilateral total hip replacement. Two measurements were made on each hip: the centre edge angle as defined by Wiberg ${ }^{17}$ and acetabular depth as defined by Murray ${ }^{6}$ using methods adapted from previous studies. ${ }^{910}$ The centre edge angle is formed by (a) a vertical line drawn from the centre of the femoral head at right angles to the line joining the two femoral head centres and (b) a line from the centre of the femoral head to the lateral edge of the acetabular roof. The centre of the femoral head was determined using a transparent paper with concentric circles drawn on it. The centre edge angles were measured using a transparent plastic protractor. Efforts were made to identify and exclude any lateral acetabular osteophytes.
Where this was unclear, we followed Wiberg's rule of taking the point where the dense acetabular 'eyebrow' finishes as the lateral margin of the roof. Acetabular depth was measured as the perpendicular distance from the deepest point of the acetabular roof to the line joining the lateral margin of the acetabular roof and the upper corner of the symphysis pubis on the same side. This method also requires careful exclusion of lateral osteophytes in identifying the margin of the roof.

Following the approach used in previous studies, an abnormal centre edge angle indicating acetabular dysplasia was defined as less than 25 degrees and an abnormal acetabular depth as less than $9 \mathrm{~mm} .{ }^{6911}$

\section{REPEATABILITY}

After training conducted by an experienced investigator (CC), both interobserver and intraobserver reliability of the two measurements of acetabular dysplasia were established between the trainer and the rater informally by the trainer (CC). The rater then carried out all of the measurements on the 253 radiographs.

\section{STATISTICAL ANALYSIS}

The distributions of demographic and body composition variables were compared between the OA and control groups. The prevalence of acetabular dysplasia in the cases of OA and controls was then calculated and the association between acetabular dysplasia and hip OA was determined using multiple logistic regression analyses. Two series of analyses were run: one series of three multiple logistic regression analyses with subjects as the unit of analysis, and one series of three multiple logistic regression analyses with hips as the unit of analysis. For the hip based analysis, hips $(n=82)$ with a summary grade of one were excluded from this analysis to maximise the contrast between the hip OA group and the control group. This yielded approximately equal numbers of hips with $\mathrm{OA}(\mathrm{n}=219)$ and without OA $(n=195)$ with complete data on acetabular dysplasia. Three analyses per series result from the fact that we had three predictor variables of interest: centre edge angle, acetabular depth, or acetabular dysplasia. Each analysis examined the association of hip OA with one of the dichotomous predictor variables of interest, with age and investigational site as covariates. Odds ratios and 95\% confidence intervals (CI) were calculated from

Table 1 Patient characteristics of the study population with no radiographic hip $O A$ and radiographic hip $O A$

\begin{tabular}{llll}
\hline Variables & $\begin{array}{l}\text { No } O A \\
(n=88)\end{array}$ & $O A(n=165)$ & $p$ Values \\
\hline Age (y) & $71.0(4.7)$ & $72.4(5.5)$ & 0.05 \\
Height (cm) & $159.4(5.5)$ & $160.3(5.9)$ & 0.24 \\
Weight (kg) & $66.1(12.2)$ & $68.3(12.9)$ & 0.19 \\
BMI & $26.1(4.9)$ & $26.6(4.7)$ & 0.42 \\
Hip pain, (past & & & \\
$\quad$ year) & $26 \%$ & $40 \%$ & 0.03 \\
Current smoker & $9 \%$ & $10 \%$ & 0.86 \\
Current oestrogen & $11 \%$ & $10 \%$ & 0.68 \\
\hline
\end{tabular}

Data shown as mean (SD). 
Table 2 Measures of acetabular dysplasia by presence of radiographic changes of hip $O A$ (subject based analysis)

\begin{tabular}{llll}
\hline & $\begin{array}{l}\text { Subjects with } \geq 1 \\
\text { grade 2-4 hip } \\
(n=158)(\%)\end{array}$ & $\begin{array}{l}\text { Subjects with grade 0 } \\
\text { hips }(n=88)(\%)\end{array}$ & OR (95\% CI) \\
\hline Centre edge angle $<25^{\circ}$ & $8(5.1)$ & $4(4.5)$ & $\begin{array}{l}1.14(0.32,4.00) \\
\mathrm{p}=0.84 \\
1.42(0.68,2.98) \\
\mathrm{p}=0.35 \\
1.27(0.64,2.51) \\
\text { Acetabular depth }<9 \mathrm{~mm}\end{array}$ \\
Acetabular dysplasia & $29(18.4)$ & $12(13.6)$ & 0.49 \\
\hline
\end{tabular}

Table 3 Measures of acetabular dysplasia by presence of radiographic changes of hip $O A$ (hip based analysis)

\begin{tabular}{|c|c|c|c|}
\hline & $\begin{array}{l}\text { Grade } 2-4 \\
(n=219)\end{array}$ & $\begin{array}{l}\text { Grade 0 } \\
(n=195)\end{array}$ & OR $(95 \% C I)$ \\
\hline Centre edge angle $\left(\right.$ mean $\left.^{\circ}, \mathrm{SD}\right)$ & $36.7(7.6)$ & $35.4(5.7)^{\star}$ & NA \\
\hline Centre edge angle $<25^{\circ}(\%)$ & $9(4.1 \%)$ & $5(2.6 \%)$ & $\begin{array}{l}1.43(0.46,4.46) \\
p=0.54\end{array}$ \\
\hline Acetabular depth (mm, SD) & $12.2(3.2)$ & $11.9(2.5)$ & NA \\
\hline Acetabular depth $<9 \mathrm{~mm}$ & $28(12.8 \%)$ & $18(9.2 \%)$ & $\begin{array}{l}1.47(0.78,2.77) \\
p=0.24\end{array}$ \\
\hline Acetabular dysplasia & $32(14.6 \%)$ & $22(11.3 \%)$ & $\begin{array}{l}1.33(0.74,2.40) \\
\mathrm{p}=.034\end{array}$ \\
\hline
\end{tabular}

${ }^{\star} \mathrm{P}<0.05, t$ test compared with grade $2-4$.

the estimated regression coefficients and their standard errors. The $5 \%$ level of statistical significance was used.

\section{Results}

Women whose pelvic radiographs were assessed for measures of acetabular dysplasia had a mean (SD) age of 72 (5.3) years, mean (SD) weight of $67.4(12.6) \mathrm{kg}$, mean (SD) height of $159.9(5.8) \mathrm{cm}$, and mean (SD) body mass index of 26.4 (4.8). Women with hip OA were significantly older than those without hip OA $(p<0.05)$ (table 1$)$. There were no other significant differences in the means of these variables between women with hip $\mathrm{OA}$ and those without.

The mean (SD) centre edge angle of 251 evaluable right and 247 evaluable left hips was 35.5 (6.5) degrees and 37.1 (6.7) degrees, respectively. Ten $(4.0 \%)$ right and six $(2.4 \%)$ left hips had an abnormal centre edge angle, or less than 25 degrees. The mean (SD) acetabular depth was 11.9 (2.9) $\mathrm{mm}$ and 12.2 (3.1) $\mathrm{mm}$ for the right and left hips, respectively. Thirty three $(13.1 \%)$ right and $22(8.9 \%)$ left hips had an abnormal acetabular depth, or less than $9 \mathrm{~mm}$. Acetabular dysplasia (abnormal centre edge angle or acetabular depth) was present in $38(15.1 \%)$ right and $26(10.5 \%)$ left hips.

Neither of the measures of acetabular dysplasia, nor the two measures combined, were significantly associated with the presence of hip OA in the subject based analyses (table 2 ) or in the hip based analyses (table 3). The mean centre edge angle was significantly greater in hips with OA compared with normal hips, but there was no difference in mean acetabular depth (table 3).

\section{Discussion}

These data do not support the hypothesis that subclinical acetabular dysplasia is associated with hip OA in elderly white women. However, we are unable to exclude the possibility that the presence of mild acetabular dysplasia is associated with a small increase in the risk of radiographic changes of hip osteoarthritis in older women.

Clinical studies have suggested that congenital and developmental diseases of the hip, including congenital dislocation/subluxation, slipped capital femoral epiphysis, and Legg-Calve-Perthe's disease, may predispose to premature hip OA..$^{5-819}$ These disorders are too uncommon to explain a large proportion of cases in the elderly, ${ }^{2}$ and it is probable that women with these conditions would present with clinical signs of hip OA at younger ages. Subclinical acetabular dysplasia has also been suggested to be a risk factor for hip OA in older adults, being present in between 25 and 40 per cent of cases in two large series. ${ }^{67}$ The hypothetical mechanism to explain this association is the presence of a subtle biomechanical abnormality, secondary to either joint incongruity or decreased joint surface area, which increases joint stresses in the superolateral acetabular rim. Indeed, Wilson and Poss ${ }^{5}$ stated that 'Deformity of the acetabulum, usually caused by childhood acetabular dysplasia, accounts for $25 \%$ to $35 \%$ of adult cases of OA of the hip, with a male-tofemale ratio of $1: 10$.'

The results of this study are similar to those of several previous studies in failing to lend support to this hypothesis. These studies did not find a significant relation between measures of mild acetabular dysplasia and radiographic hip OA in elderly women ${ }^{10}$ or in men. ${ }^{911}$ The number of cases of hip OA in these studies was small and statistical power to detect an association with dichotomous measures of an abnormally shallow acetabulum may have been limited. Nevertheless, the mean centre edge angle and acetabular depth were inversely associated with the severity of joint space narrowing, ${ }^{910}$ and tended to be greater in hips with OA compared with normal hips, ${ }^{9-11}$ the opposite of the hypothesised relation. Indeed, in the two studies that examined this ${ }^{911}$ the prevalence of abnormal centre edge angle and acetabular depth was lower in hips with OA compared with normal hips.

Our study has several limitations. While we had a power of $80 \%$ (alpha $=0.05$, two sided) to detect very small differences in the mean centre edge angle (1.8 degrees) and acetabular depth $(0.8 \mathrm{~mm})$ between hips with and without $\mathrm{OA}$, our power to detect associations between some of the dichotomous measures of hip dysplasia and OA was limited. For example, in hip based analyses our power to detect a doubling of the prevalence of mild dysplasia in hips with OA (corresponding to an odds ratio of approximately 2) was $18 \%$ for abnormal centre edge angle, but was $72 \%$ for abnormal acetabular depth and $83 \%$ for acetabular dysplasia (alpha $=0.05$, two sided for all calculations). Our power was somewhat less for subject based analyses, but odds ratios were similar for both analyses. If the true magnitude of the association between abnormal hip geometry and OA was what we actually observed (odds ratios of 1.3 to 1.5 ) our study 
would have required a much larger sample size to detect such an association. Nevertheless, the clinical significance of an association of this magnitude is uncertain. It is possible that other aspects of abnormal hip geometry not assessed in this or previous studies are risk factors for hip OA in the elderly. ${ }^{20}$ Hip OA may change femoral and acetabular geometry, increasing both the acetabular depth and centre edge angle. ${ }^{10}$ Prospective studies are needed that measure geometry before the development of hip OA. ${ }^{19}$ Total hip arthroplasty for treatment of early hip OA would have prevented evaluation of hip geometry and hip OA for this study. As we studied only elderly people, our study cannot resolve whether mild acetabular dysplasia is an important cause of early hip OA in younger women.

This study differs from previous reports that have examined this possible association. The subjects in this study were volunteers selected from population based lists who were participating in a longitudinal cohort study of factors associated with osteoporotic fractures. In addition, this study used an efficient nested case control study design with a random sample of pelvic radiographs enriched with cases of hip OA. This contrasts with previous studies, which examined the association of acetabular dysplasia with hip OA in patients with medical conditions that required an intravenous urogram wherein there was a low prevalence, and therefore few cases, of hip OA ${ }^{9-11}$ and with studies of surgical case series of patients with hip $\mathrm{OA}$ and no comparison group. ${ }^{5-8}$ Mean values for centre edge angle, and the prevalence of abnormal values, in our study were similar to that of previous studies..$^{9-1121}$ Values for acetabular depth, and the prevalence of abnormals, were similar to that in Hong Kong Chinese men, ${ }^{11}$ but less than observed in British women ${ }^{10}$ and men. ${ }^{9}$

In summary, we failed to detect an association of subclinical acetabular dysplasia with hip OA in elderly white women. Our results, and those of previous studies, suggest that mild acetabular dysplasia is not a common or important cause of hip OA in the elderly population.

This research was supported by a grant from the NIH (1-R01AG05407, 1-R01-AR40431) and an educational grant from the Northern California Arthritis Foundation and the Rosalind Russell Arthritis Center.
1 Scott JC, Hochberg MC. Epidemiologic insights into the pathogenesis of hip osteoarthritis. In: Hadler NM, ed. Clinical concepts in regional musculoskeletal illness. Orlando: Grune \& Stratton, 1987: 89-108.

2 Felson DT. Epidemiology of hip and knee osteoarthritis. Epidemiol Rev 1988;10:1-28.

3 Tepper S, Hochberg MC. Factors associated with hip osteoarthritis: data from the First National Health and Nutrition Examination Survey. Am J Epidemiol 1993;295:11926.

4 Heliövaara $M$, Mäkela $M$, Impivaara $\mathrm{O}$, Knett $\mathrm{P}$, Aro Maa A, Sievers K, et al. Association of overweight, trauma, and workload with coxarthrosis. Acta Orthop Scand 1993; 64:513-8.

5 Wilson MG, Poss R. Osteoarthritis of the hip. In: Moskowitz RW, Howell DS, Goldberg VM, Mankin HJ, eds. Osteoarthritis: diagnosis and medical/surgical management. Philadelphia: W B Saunders, 1992: 621-50.

6 Murray RO. The aetiology of primary osteoarthritis of the hip. Br J Radiol 1965;38:810-24.

7 Stulberg SD, Cordell LD, Harris WH, Ramsey PL, MacEwen GD. Unrecognized childhood hip disease: a major cause of idipathic osteoarthritis of the hip. Hip 1975;3:212-30.

8 Harris WH: Etiology of osteoarthritis of the hip. Clin Orthop 1986;213:20-33.

9 Croft P, Cooper C, Wickham C, Coggon D. Osteoarthritis of the hip joint and acetabular dysplasia. Ann Rheum Dis 1991;50:308-10.

10 Smith RW, Egger P, Coggon D, Cawley MID, Cooper C. Osteoarthritis of the hip joint and acetabular dysplasia in women. Ann Rheum Dis 1995;54:179-81.

11 Lau E, Lin F, Lam D, Silman A, Croft P. Hip osteoarthritis and dysplasia in Chinese men. Ann Rheum Dis 1995; 54:965-9.

12 Cummings SR, Nevitt MC, Browner WS, Stone K, Fox $\mathrm{KM}$, Ensrud K, et al. Risk factors for hip fracture in white women. N Engl J Med 1995;332:767-73.

13 Lane NE, Nevitt MC, Genant HK, Hochberg MC. Reliability of new indices of radiographic osteoarthritis of the hand and hip and lumbar disc degeneration. J Rheumatol 1993; 20:1911-8.

14 Nevitt MC, Lane NE, Scott JC, Hochberg MC, Pressman AR, Genant HK, et al. Radiographic osteoarthritis of the hip and bone mineral density. Arthritis Rheum 1995; 38:907-16.

15 Scott JC, Nevitt MC, Pressman AR, Lane NE, Hochberg MC. Association of radiographic features of hip osteoarthritis with pain. [Abstract]. Arthritis Rheum 1992;35 (suppl):S81.

16 Scott JC, Nevitt MC, Pressman AR, Lane NE, Hochberg MC, Genant HK. Association of functional measures with radiographically defined hip osteoarthritis. [Abstract]. Arthritis Rheum 1993;36 (suppl):S235.

17 Hochberg MC, Lane NE, Pressman AR, Genant HK, Scott JC, Nevitt MC. The association of radiographic changes of osteoarthritis of the hand and hip in elderly women. J Rheumatol 1995;22:2291-4.

18 Wiberg G. A measuring method for distinguishing between a normal and a maldeveloped acetabulum. Acta Chir Scand 1939;83 (suppl 1):28-38.

19 Cooperman D, Wallenstein R, Stulberg S. Acetabular dysplasia in the adult. Clin Orthop 1983;175:79-85.

20 Wedge JH, Wasylenko MJ, Houston CS. Minor anatomic abnormailities of the hip joint persisting from childhood and their possible relationship to idiopathic osteoarthrosis. Clin Orthop 1991;264:122-8.

21 Fredensborg N. The CE angle of normal hips. Acta Orthop Scand 1976;47:403-5. 